\title{
Nanocomposite hydrogel as a template for synthesis of mono and bimetallic nanoparticles
}

\author{
D. Varade $^{1}$, K. Haraguchi ${ }^{2}$ \\ ${ }^{1}$ School of Engineering and Applied Science, Ahmedabad University, GICT Building, Central Campus, Navrangpura, \\ 380009 Ahmedabad, Gujarat, India \\ ${ }^{2}$ Department of Applied Molecular Chemistry, College of Industrial Technology, Nihon University, 275-8575 Chiba, \\ Japan
}

Received 9 April 2018; accepted in revised form 6 June 2018

\begin{abstract}
Nanocomposite hydrogels (NC gels) with polymer-clay network assemblies are useful in a number of applications because of their unique properties and characteristics. Herein, we describe a distinctive strategy for the preparation of discrete monometallic ( $\mathrm{Ag}, \mathrm{Au}$, and $\mathrm{Pd}$ ) and bimetallic (Pt-Pd, $\mathrm{Au}-\mathrm{Pd})$ nanoparticles that uses a nanocomposite hydrogel composed of a polymer-clay network. Thermoresponsive NC gels were synthesized by the in-situ free-radical polymerization of $N$ isopropylacrylamide in the presence of clay (synthetic hectorite) nanosheets (CNSs). Since CNSs have strong affinities for metals ions that facilitate the concentration of metal precursors around them, the reduction of metal ions by ascorbic acid in NC gels provides well-dispersed, non-aggregated spherical monometallic and bimetallic nanoparticles (NPs) that are strongly immobilized within the polymer-clay network. The resulting hybrid NP-NC gels, which contain monometallic or bimetallic NPs, exhibit high catalytic activities for the hydrogenation of nitrophenol to aminophenol. The combination of well-defined metal NPs and mechanically tough NC gels opens up new possibilities for the design of environmentally friendly and sustainable functional NP-NC-gel materials.
\end{abstract}

Keywords: nanomaterials, nanocomposites, bimetallic nanoparticles, polymer gels, polymer-clay network

\section{Introduction}

Nanocomposite hydrogels (NC gels) with unique organic-inorganic (polymer-clay) network assemblies have drawn great interest as excellent hydrogels that can overcome the severe drawbacks associated with conventional chemically cross-linked hydrogels [15]. Specifically, NC gels composed of poly( $N$-isopropylacrylamide) (PNIPAM) and exfoliated inorganic clay, such as synthetic hectorite, have displayed extraordinary optical, mechanical, and swelling/ deswelling properties [3]. In addition, $\mathrm{NC}$ gels can be prepared effortlessly at ambient temperature in an aqueous system $[3,6]$, to provide a variety of shapes, sizes, and surface morphologies, along with novel surface characteristics such as unique sliding friction, ultrahigh hydrophobicities, cell harvesting, self-healing, and contractive-force-generating capabilities [3, $7,8]$, which makes them useful in many new applications. $\mathrm{NC}$ gels have also contributed to a wide range of developments as new stimuli-responsive soft and wet materials [9-12]. These observations provide encouragement for the further extension of NC-gel templates to bulk materials that encompass nanosized metal configurations distributed within their structures; these metal-imbedded gels are prospective materials for use in catalysts and sensors, among other applications [12].

Progress in the development of novel methods for the synthesis of metal nanoparticles (NPs) continues to be motivated by prospective applications that are 
facilitated by their unique properties [13, 14]. Numerous studies have been conducted into strategies for the preparation of noble-metal-based NPs, including NP-hydrogel fusions that are formed by exploiting the benefits of the interspatial regions enclosed by crosslinking points as nanoreactors or nanocarriers [15-20]. An NC gel consisting of a polymer-clay matrix is appropriate for concentrating metal ions because the exfoliated clay contains effective chelation sites. Recently, we reported the exploitation of NC gels as templates for the in-situ preparation of Pt NPs, which we attributed to the mild reducing ability of clay [12]. Consequently, NC gels that encompass metal ions are encouraging as 'reactors' for the well-regulated production of metal NPs, where strong interactions between the clay and metals essentially play imperative roles that govern the evolution of inorganic NPs [21]. It is worth noting that low-cost inorganic clay is deliberately used in a number of materials applications [22-24]. In the present study, we focused on the syntheses of monometallic (Ag, $\mathrm{Au}$, and $\mathrm{Pd}$ ) and bimetallic (Pt-Pd and $\mathrm{Au}-\mathrm{Pd}$ ) NPs within NC gels by carrying out the reduction of NC-gel-absorbed metal ions within a hydrogel matrix. This resulted in the creation of small, uniform, and stable NPs within the NC gel. The ensuing NP-hybrid-NC-gel materials, consisting of NPs effectively immobilized in polymer-clay networks, were then used to catalytically reduce nitrophenol.

\section{Experimental section}

\subsection{Materials}

As an inorganic clay, synthetic hectorite 'Laponite XLG' (Rockwood, Ltd., UK;

$\left[\mathrm{Mg}_{5.34} \mathrm{Li}_{0.66} \mathrm{Si}_{8} \mathrm{O}_{20}(\mathrm{OH})_{4}\right] \mathrm{Na}_{0.66}$ : layer size $=30 \mathrm{~nm}$ diameter $\times 1 \mathrm{~nm}$ thick; cation-exchange capacity $=$ $104 \mathrm{~m}$ equiv./100 g) [2] was used after washing with ethanol/water $(90 / 10 \mathrm{w} / \mathrm{w})$ and freeze-drying. $N$-Isopropylacrylamide (NIPAM) (Kohjin Co., Japan) was purified by recrystallization from $2: 1(\mathrm{w} / \mathrm{w})$ toluene: $n$ hexane, followed by drying under vacuum at $40^{\circ} \mathrm{C}$. Potassium persulfate (KPS) and $N, N, N^{\prime}, N^{\prime}$-tetramethylethylenediamine (TEMED) (Tokyo Chemical Industry Co. Ltd., Japan) were used as initiator and accelerator, respectively, during the synthesis of the NC gels. Analytical grade ascorbic acid, silver nitrate $\left(\mathrm{AgNO}_{3}\right)$, sodium tetrachloroaurate(III) $\left[\mathrm{Na}\left(\mathrm{AuC}_{14}\right) \cdot 2 \mathrm{H}_{2} \mathrm{O}\right]$, potassium tetrachloroplatinate(II) $\left(\mathrm{K}_{2} \mathrm{PtCl}_{4}\right)$, 4-nitrophenol, and sodium borohydride $\left(\mathrm{NaBH}_{4}\right)$ were purchased from Wako Pure Chemical
Industries, Ltd., Japan, and potassium tetrachloropalladate(II) $\left(\mathrm{K}_{2} \mathrm{PdCl}_{4}\right)$ was obtained from Alfa Aesar. All reagents were used without further purification, unless otherwise stated. Ultrapure water, supplied by a PURIC-MX system (Organo Co., Japan), was used for all experiments.

\subsection{NC gel synthesis}

A nanocomposite hydrogel with a PNIPAM-clay network is referred to as an 'NCn gel', where $n$ refers to the clay content $\left(\mathrm{C}_{\text {clay }}=n \mathrm{~mol} \%\right.$ in the reaction solution), by analogy with terminology used in previous papers [3]. The procedure for the syntheses of the NC gels in this study is the same as that reported previously. Briefly, the NC5 gel was synthesized from a transparent aqueous solution of inorganic clay (Laponite XLG, $0.76 \mathrm{~g}$ ), monomer (NIPAM, $2.26 \mathrm{~g}: 1 \mathrm{M})$, and water $(19 \mathrm{~mL})$. An accelerator (TEMED, $16 \mu \mathrm{L}$ ) and an aqueous solution of the initiator (KPS, $0.02 \mathrm{~g}, 1 \mathrm{~mL}$ ) were added while stirring at $1{ }^{\circ} \mathrm{C}$. Free radical polymerization was conducted in a water bath $\left(20^{\circ} \mathrm{C}\right)$ for $24 \mathrm{~h}$. The $\mathrm{NC}$ gel was synthesized in glass tube with inner diameters of $5.5 \mathrm{~mm}$. Oxygen was excluded from the water and the system throughout these syntheses. The resulting transparent NC5 gel was rinsed several times with Milli-Q water.

\subsection{Synthesis of mono and bimetallic nanoparticles within the $\mathrm{NC}$ gel}

Samples of the as-prepared NC5 gel $(\varnothing 5.5 \mathrm{~mm} \times$ $12 \mathrm{~mm}$ ), after washing with water, were immersed in individual aqueous solutions $(10 \mathrm{~mL}, 10 \mathrm{mM})$ of various metal precursors $\left(\mathrm{AgNO}_{3}, \mathrm{NaAuCl}_{4}, \mathrm{~K}_{2} \mathrm{PdCl}_{4}\right.$, $\mathrm{K}_{2} \mathrm{PtCl}_{4}+\mathrm{K}_{2} \mathrm{PdCl}_{4}$, and $\left.\mathrm{NaAuCl}_{4}+\mathrm{K}_{2} \mathrm{PdCl}_{4}\right)$ and incubated at $25^{\circ} \mathrm{C}$ for $24 \mathrm{~h}$. The resulting swollen $\mathrm{NC}$ gels were quickly rinsed with Milli-Q water within several seconds and placed separately into freshly prepared solutions of ascorbic acid $(10 \mathrm{~mL}, 100 \mathrm{mM})$ at $25^{\circ} \mathrm{C}$ for $24 \mathrm{~h}$, which reduced the metal ions within the NC-gel matrix.

\subsection{Catalytic reduction of $p$-nitrophenol}

A $0.1 \mathrm{~g}$ sample of an NP-containing NC5 gel (NPNC5 gel) was added directly to $1 \mathrm{~mL}$ of a $0.2 \mathrm{mM}$ aqueous solution of $p$-nitrophenol and $1 \mathrm{mM} \mathrm{NaBH}_{4}$ in a $1 \mathrm{~mm}$ quartz cuvette. Time-resolved UV-vis absorption spectra of the solution above the hydrogel were acquired at room temperature using a Hitachi U-4100 UV-vis double-beam spectrometer. 


\subsection{Materials characterization}

Transmission-electron microscopy (TEM) images were obtained for ultrathin films $(60 \mathrm{~nm})$ of the dried NP-NC5 gels using a high-resolution field-emission transmission-electron microscope (JEM-2200TFE, JEOL) operating at $200 \mathrm{kV}$. Energy-dispersive X-ray spectroscopy (EDS) was performed using a scanning transmission-electron-microscopy (STEM) detector fitted to the JEM-2200TFE system. XRD patterns were obtained using a Rigaku SmartLab X-ray diffractometer with monochromated $\mathrm{Cu}-\mathrm{K} \alpha$ radiation (40 kV, $100 \mathrm{~mA})$.

\section{Results and discussion}

The objective of the proposed synthesis procedure was the creation of uniform and well-dispersed monometallic (Ag, $\mathrm{Au}$, and $\mathrm{Pd}$ ) and bimetallic (Pt-Pd and Au-Pd) NPs within the NC gel matrix that are exceedingly functional and have high thermal and dispersion stabilities. The NC5 gel, which is composed of PNIPAM and synthetic hectorite $\left(\mathrm{C}_{\text {clay }}=5 \mathrm{~mol} \%\right)$, was initially prepared according to the procedure reported previously (Figure 1a) [3]. The NC5 gel was obtained as a uniform, transparent, and mechanically tough hydrogel, and exhibited notable tensile mechanical properties (tensile strength $=120 \mathrm{kPa}$, elongation at break $=1010 \%$ ), a considerable degree of swelling in water $\left(W_{\text {gel }} / W_{\text {dry }}=50\right.$ at $\left.20^{\circ} \mathrm{C}\right)$, and a well-defined thermo-responsive transition at $34^{\circ} \mathrm{C}$ (lower critical solution temperature: LCST) derived from the coil-to-globule transition of the constituent PNIPAM.

NC5-gel samples were separately immersed in aqueous solutions of $\mathrm{Ag}, \mathrm{Au}$, and Pd-metal precursors $(10 \mathrm{mM})$, after which they were maintained in the

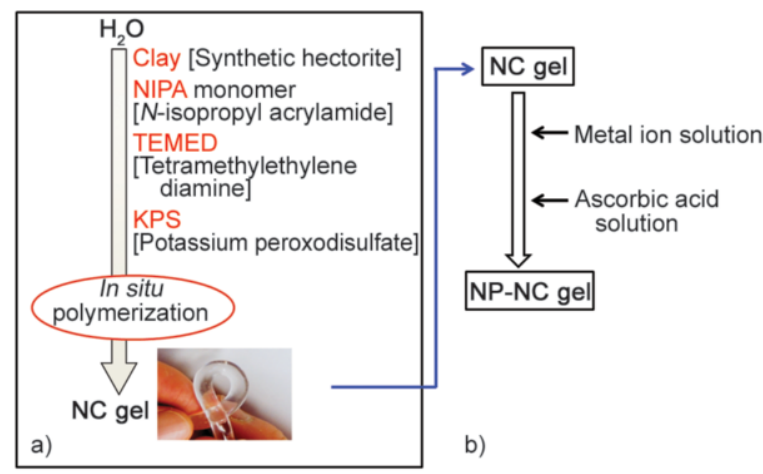

Figure 1. (a) Protocol for the synthesis of an NC gel under ambient conditions. (b) Incorporation of metal NPs into the NC gel by immersion in an aqueous metal-precursor solution followed by reduction by immersion in aqueous ascorbic acid. dark at $25^{\circ} \mathrm{C}$ for $24 \mathrm{~h}$, during which time they swelled (Figures $1 \mathrm{~b}$ and $2 \mathrm{a}$ ).

The resulting swollen NC gels were quickly rinsed with Milli-Q water for a few seconds and immersed in freshly prepared aqueous solutions of ascorbic acid $(100 \mathrm{mM})$; reductions of the corresponding metal ions were conducted within the NC-gel matrices while standing for $24 \mathrm{~h}$ at $25^{\circ} \mathrm{C}$ (Figure 2b).

Data on the sizes and shapes of the NPs were obtained by transmission-electron microscopy (TEM). Figure 3a shows a high-angle annular dark-field scanning TEM (HAADF-STEM) image of an ultrathin section of the dried Ag/NP-NC gel, which reveals that small and well-defined $\mathrm{Ag} / \mathrm{NPs}$, with diameters of $40-50 \mathrm{~nm}$, are distributed throughout the NC-gel sample. The EDS image shown in Figure $3 \mathrm{~b}$ confirms the formation of $\mathrm{Ag} / \mathrm{NPs}$ in the $\mathrm{NC}$ gel. The formation of uniform and well-dispersed $\mathrm{Ag} / \mathrm{NPs}$ in the $\mathrm{NC}$ gel is attributed to the exfoliated clay nanosheets (CNSs)
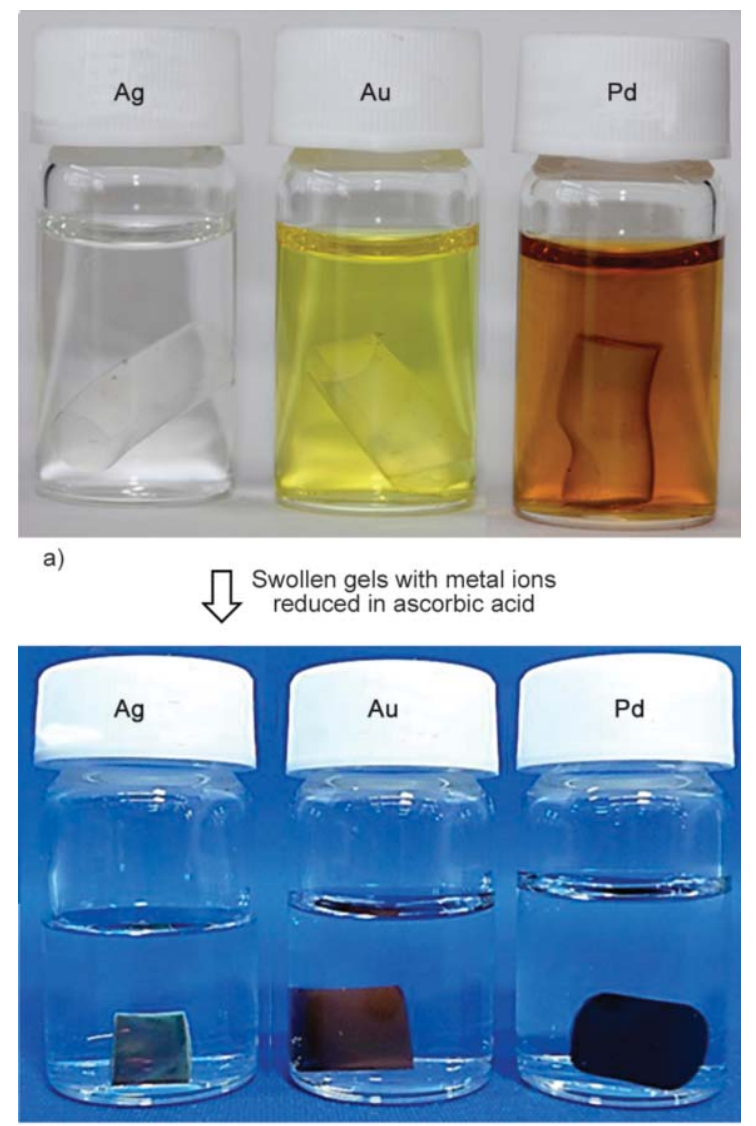

b)

Figure 2. Optical images depicting the preparation of NP$\mathrm{NC}$ gels (where NP $=\mathrm{Ag}, \mathrm{Au}$, and Pd NPs). (a) Absorption of $\mathrm{Ag}, \mathrm{Au}$, or $\mathrm{Pd}$ metal ions into the $\mathrm{NC}$ gel through the swelling process $\left(24 \mathrm{~h}, 25^{\circ} \mathrm{C}\right)$. (b) Each swollen NC gel was immersed in ascorbic acid solution $(100 \mathrm{mM})$, which resulted in the reduction of the metal ions within the $\mathrm{NC}$ gel. 


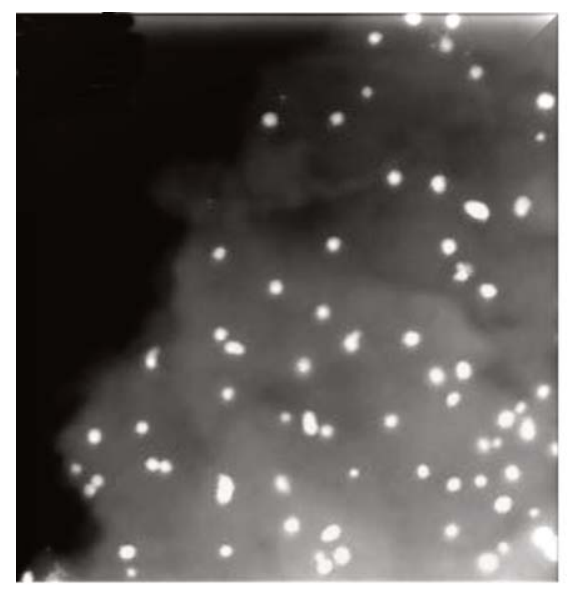

a)

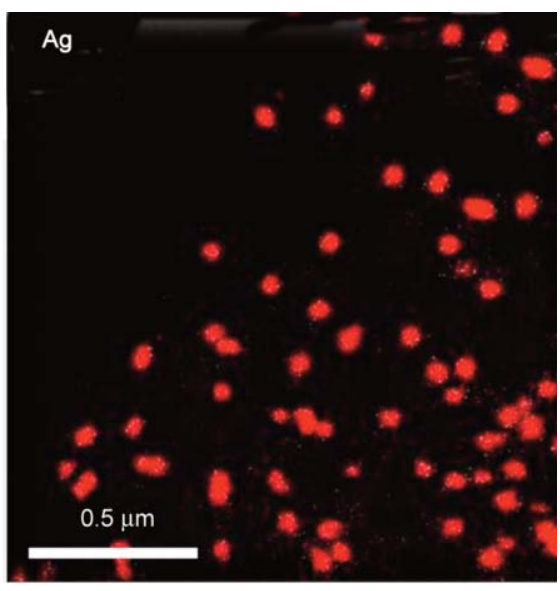

b)

Figure 3. (a) HAADF-STEM image of Ag/NPs in the NC gel matrix. (b) EDS image showing well-defined Ag NPs.

that are uniformly dispersed in the $\mathrm{NC}$ gel and form a three-dimensional polymer-clay network; reduction occurs effectively near the clay nanosheet (CNS) surface, resulting in a new nanostructured, polymerclay-NP hybrid material.

Similarly, Figure 4a shows a HAADF-STEM image of an ultrathin section of the dried Au/NP-NC gel. Well-defined $\mathrm{Au}$ NPs, with an average diameter of $85 \mathrm{~nm}$, are observed to be well dispersed over the NC gel sample, as confirmed by the EDS image displayed in Figure 4b. A Pd/NP-NC gel was also prepared in the same manner (figure not shown).

Figure 5 shows wide-angle X-ray diffraction (XRD) patterns of the $\mathrm{Ag}, \mathrm{Au}$, and Pd NPs prepared within the NC-gel matrices. In all cases, multiple diffraction peaks that are indexed to the $f c c$ lattices of the corresponding $\mathrm{Ag}, \mathrm{Au}$, and $\mathrm{Pd}$ crystals were observed.

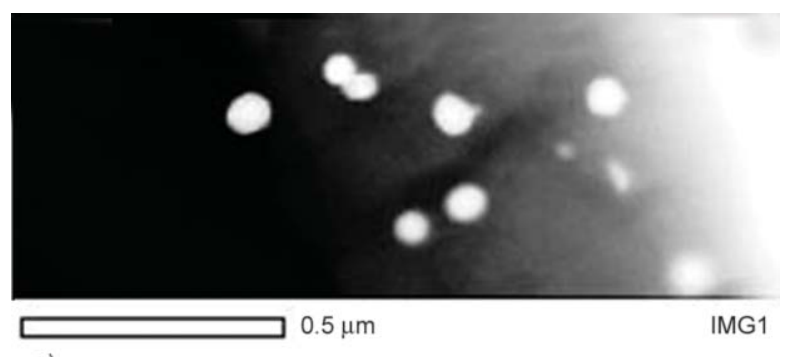$$
\text { a) }
$$

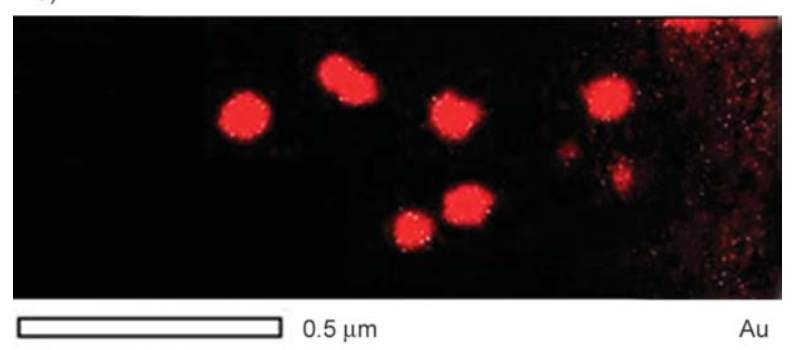

b)

Figure 4. (a) HAADF-STEM image of Au NPs in the NC gel matrix. (b) EDS image showing well-defined Au NPs.
To discover the full potential of this approach, we also synthesized bimetallic NPs, such as Pt-Pd and AuPd NPs, in a similar fashion, except that two metal precursors were used. In the case of the Pt-Pd NPs (Figure 6), the as-prepared $\mathrm{NC}$ gel was immersed in a mixed solution of the metal precursors $\left(\mathrm{K}_{2} \mathrm{PtCl}_{4}+\right.$ $\mathrm{K}_{2} \mathrm{PdCl}_{4}: 5 \mathrm{mM}$ each) at $25^{\circ} \mathrm{C}$ for $24 \mathrm{~h}$. The swollen $\mathrm{NC}$ gel was then quickly washed with Milli-Q water and immersed in a fresh aqueous solution of ascorbic acid $(100 \mathrm{mM})$ at $25^{\circ} \mathrm{C}$ for $24 \mathrm{~h}$ in order to reduce the metal ions within the NC-gel matrix to bimetallic Pt-Pd NPs. The morphologies of bimetallic NPs are difficult to control by conventional methods; consequently precisely formulating these NPs can be challenging.

Figure 6a displays a representative TEM image of an ultrathin section of the dried Pt-Pd/NP-NC gel, which reveals the successful formation of well-defined Pt-Pd NPs, with diameters of 50-55 nm that well distributed over the NC gel sample. The highresolution (HR) TEM image (Figure 6b) displays clearly visible lattice fringes that provide evidence

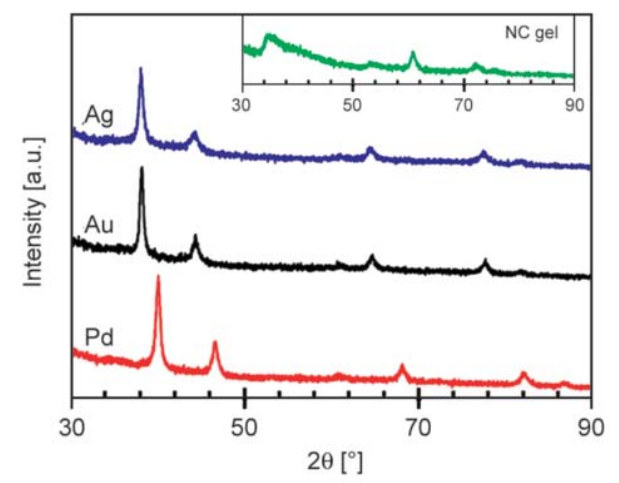

Figure 5. Wide-angle XRD patterns of dried $\mathrm{Ag}, \mathrm{Au}$, and Pd/NP-NC5-gel powders. An inset figure shows the XRD pattern for pure dried NC5 gel. 


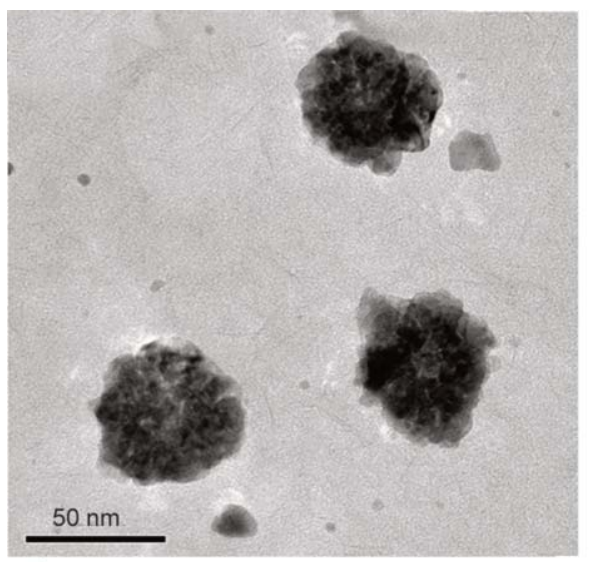

a)

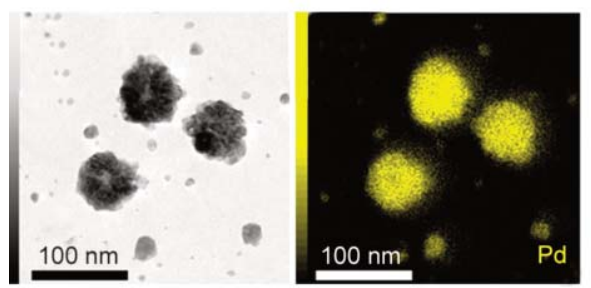

c)

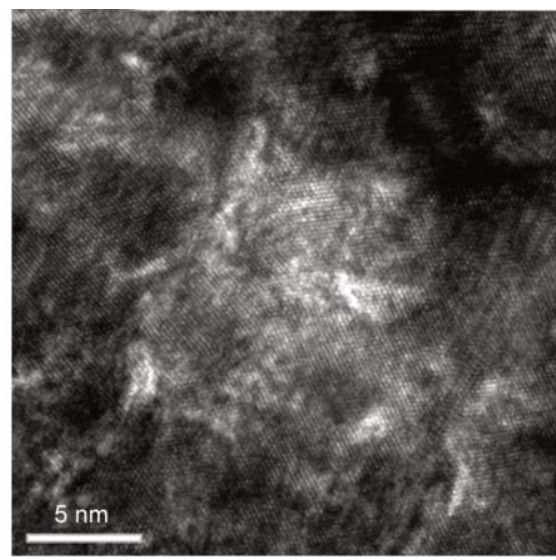

b)

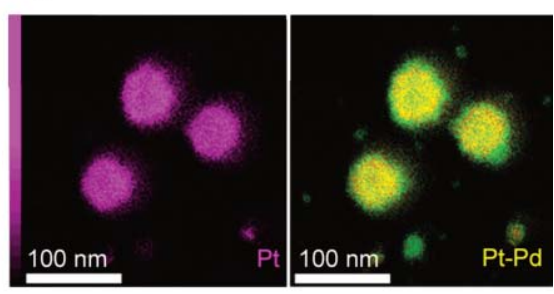

e)

f)

Figure 6. Bimetallic Pt-Pd NPs prepared in the NC5 gel matrix. (a) Low-magnification TEM image. (b) HRTEM image. (c) HAABF-STEM image. (d) Pd EDS map. (e) Pt EDS map. (f) Overlay of (d) and (e) that reveals the presence of finely distributed $\mathrm{Pt}$ and $\mathrm{Pd}$.

for the formation of crystalline NPs. Figure $6 \mathrm{c}$ shows a high-angle annular bright-field STEM (HAABFSTEM) image, while the corresponding EDS maps (Figures 6d-6f) reveal the presence of uniformly composed Pt and Pd bimetallic NPs. These results indicate that the formation of bimetallic Pt-Pd NPs is the result of nucleation control and the prevention of mutual metal cluster-cluster aggregation in the presence of the network CNSs. The exfoliated CNSs within the NC gel play very important roles in controlling the concentrations of the Pt and Pd ions. The subsequent adsorption of ascorbic acid induces the controlled reduction of metal ions near the CNS surfaces, where the NC gel matrix helps to spontaneously separate the deposited $\mathrm{Pd}$ and $\mathrm{Pt}$, resulting in the formation of bimetallic Pt-Pd NPs and the prevention of their random growth. In the absence of the clay, i.e., in case of a conventional chemically cross-linked PNIPAM gel, the reduced Pt and Pd metals ions form large inhomogeneous particles devoid of well-defined structures. Hence, we infer that the presence of the CNS is pivotal for the fabrication of bimetallic NPs; achieving this outcome within a polymer network is otherwise very challenging.

Bimetallic Au-Pd NPs were also synthesized by the methodology described above. The TEM image of an ultrathin section of the dried Au-Pd/NP-NC gel
(Figure 7a) reveal well-defined Au-Pd NPs, and the HR-TEM image (Figure 7b) exhibits visible lattice fringes that are evidence of the formation of crystalline bimetallic NPs. Figure 7c shows a HAABFSTEM image and the corresponding EDS overlay map is shown in Figure 7d, which reveals a uniform $\mathrm{Au}$ and $\mathrm{Pd}$ elemental composition.

The as-prepared monometallic and bimetallic NPs were highly stable within the NC gel matrix. Representative optical images of the Pd/NP-NC and Pt$\mathrm{Pd} / \mathrm{NP}-\mathrm{NC}$ gels immersed in water for $72 \mathrm{~h}$ reveal no signs of leaching; as the surrounding water remains clear (Figure 8). Similar observations were made even at a high temperature $\left(50^{\circ} \mathrm{C}\right)$, above the LCST, where both NP-NC gels underwent shrinkage due to the coil-to-globule transition of the PNIPAM in each gel. This indicates that, once formed, the metal NPs remain intact within the $\mathrm{NC}$ gel; this property is extremely important for NP-NC gel applications.

To evaluate their catalytic activities, we tested the abilities of the Pd/NP-NC and Pt-Pd/NP-NC gels to catalyze the reduction of aqueous 4-nitrophenol with $\mathrm{NaBH}_{4}$, as a model system (Figure 9a). The lightyellow color of the 4-nitrophenol solution immediately changed to an intense yellow upon addition of $\mathrm{NaBH}_{4}$, due to the formation of the 4-nitrophenolate ion. $\mathrm{NaBH}_{4}$ solution itself is not capable of reducing 


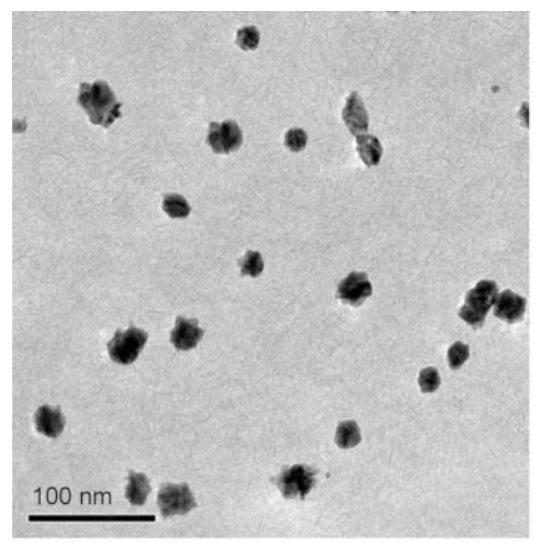

a)

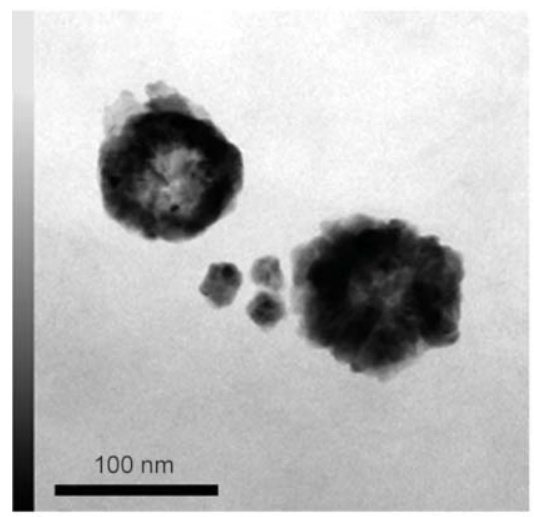

c)

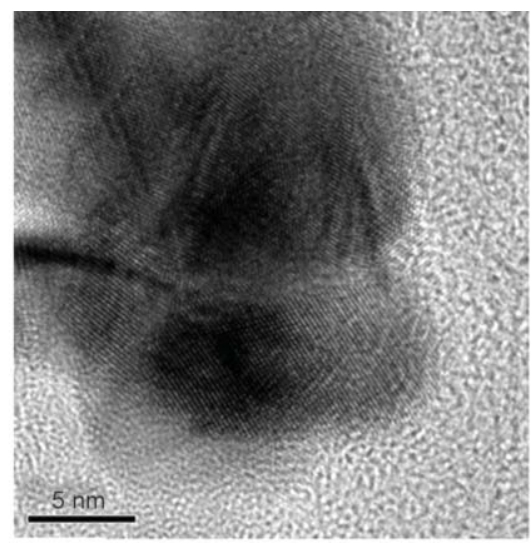

b)

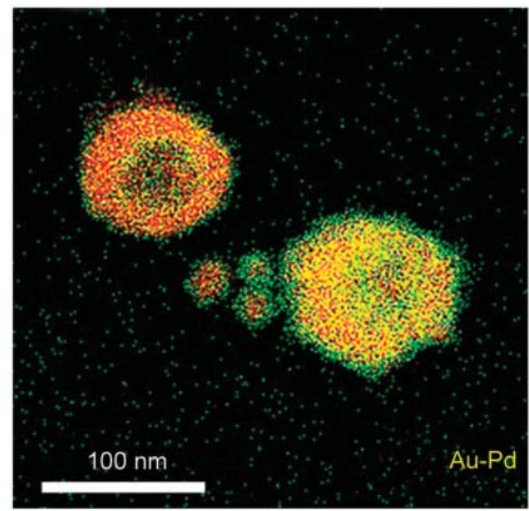

d)

Figure 7. Bimetallic Au-Pd NPs prepared in the NC5 gel matrix. (a) Low-magnification TEM image. (b) HRTEM image. (c) HAABF-STEM image. (d) EDS Au-Pd overlay map that reveals the presence of finely distributed Au and Pd.

4-nitrophenol in the absence of a catalyst, and its absorption band at around $400 \mathrm{~nm}$ remained unchanged even after $90 \mathrm{~min}$ (Figure 9b).

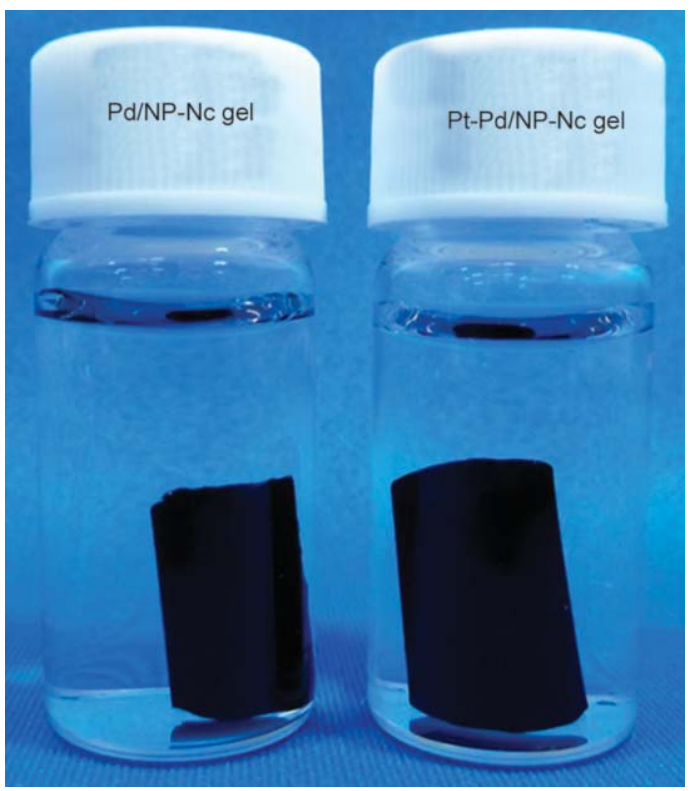

Figure 8. Optical images demonstrating a lack of nanoparticle leaching from the $\mathrm{Pd} / \mathrm{NP}-\mathrm{NC}$ and $\mathrm{Pt}-\mathrm{Pd} / \mathrm{NP}$ $\mathrm{NC}$ gels following immersion in pure water for $72 \mathrm{~h}$ under ambient conditions.
However, the presence of the Pd/NP-NC gel or $\mathrm{Pt}-\mathrm{Pd} / \mathrm{NP}-\mathrm{NC}$ gel $(0.1 \mathrm{~g})$ catalyzed the reduction of 4-nitrophenol, and the strong ultraviolet (UV) absorption peak at $400 \mathrm{~nm}$ diminished over 25 and $15 \mathrm{~min}$, respectively, as displayed in Figures 9c and $9 \mathrm{~d}$, indicating high catalytic activity. While the reduction of 4-nitrophenol was very slow in the presence of $\mathrm{NaBH}_{4}$ alone, rapid reduction was observed in the presence of each NP-NC gel. It is highly likely that other useful reactions are also accelerated by the action of these NP-NC gels; we believe that these materials will be applicable to a wide range of catalysis processes.

The proposed mechanism that underpins the formation of the mono and bimetallic NPs in the NC-gel matrix is shown in Figure 10. As depicted, when the $\mathrm{NC}$ gel is immersed in the aqueous metal-precursor solution, the metal ions penetrate the NC gel and interact with the silanol groups $(\mathrm{Si}-\mathrm{OH})$ on the $\mathrm{CNS}$ surfaces to form complexes, which results in the concentration of metal ions around the CNS surfaces of the polymer-clay network. The resulting swollen $\mathrm{NC}$ gel imbedded with metal ions is then immersed 


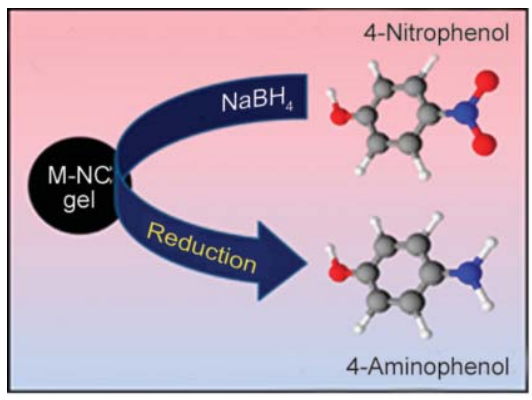

a)

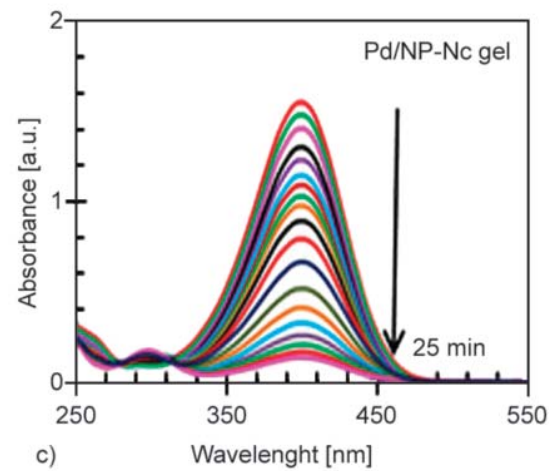

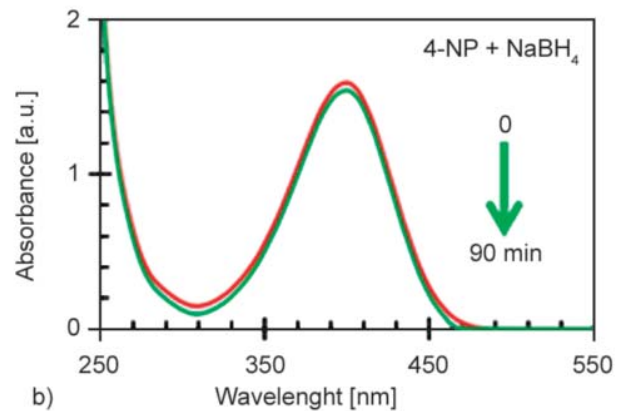

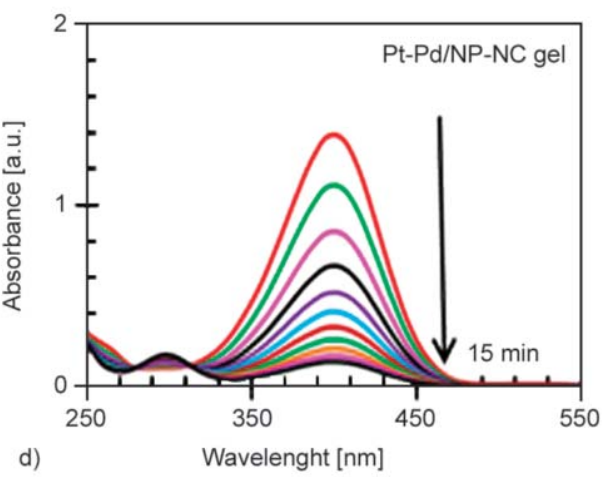

Figure 9. (a) Model reaction depicting the catalytic conversion of 4-nitrophenol into 4-aminophenol by an NP-NC gel. (b) The strong UV absorption peak at $400 \mathrm{~nm}$ corresponds to the nitrophenolate ion. However no change in the reaction occurs in the absence of a catalyst. Catalytic reduction of 4-nitrophenol by $\mathrm{NaBH}_{4}$ occurred in the presence of (c) the Pd/NP-NC gel (0.1 g), where the reaction was complete in $25 \mathrm{~min}$, and (d) the Pt-Pd/NP-NC gel (0.1 g), where the reaction was complete in $15 \mathrm{~min}$.

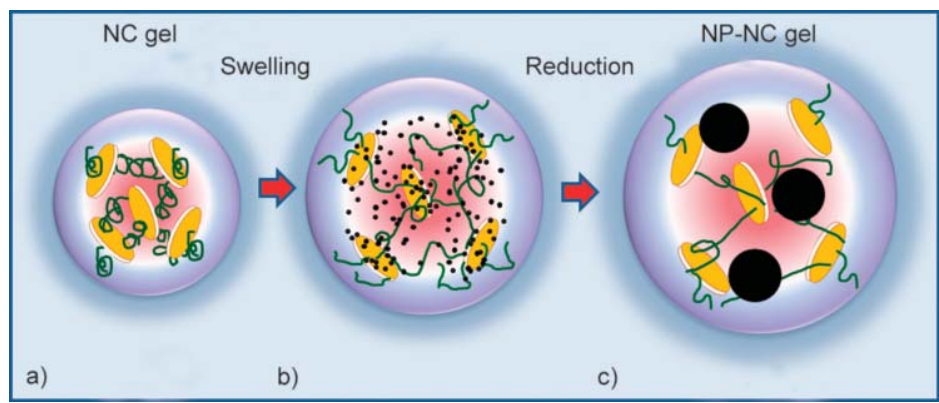

Figure 10. Depicting the formation of the hybrid NP-NC gel: (a) metal ions penetrate into the NC gel, (b) metal ions interact with the silanol groups on the clay surface, and (c) metal NPs are formed by the ascorbic-acid reduction of ions, which are subsequently trapped near the clay surface.

in the freshly prepared ascorbic acid solution, which results in the subsequent reduction of metal ions near the CNS surface and the creation of well-defined metal NPs that remain intact in the polymer-clay network matrix of the NP-NC gel. Thus, the CNSs play important roles in controlling the dispersion and the stabilization of metal NPs. Polymer gels without clay are not only very weak and brittle but also difficult to synthesize well dispersed size controlled metal NPs. Also, we have used ascorbic acid here for the slow and controlled synthesis of metal NPs as it is mild reducing agent compared to $\mathrm{NaBH}_{4}$. Moreover, we had tried to reduce the metal ions in $\mathrm{NC}$ gel using $\mathrm{NaBH}_{4}$ but we found lots of bubbling around
$\mathrm{NC}$ gel as soon as we dip the metal-ion swollen NC gel in $\mathrm{NaBH}_{4}$ solution. Also the mechanical strength of NC gel goes down. This clearly indicates that $\mathrm{NaBH}_{4}$ disturbs the structure of $\mathrm{NC}$ gel and also it does not allow controlled reduction of metal NPs within $\mathrm{NC}$ gel.

\section{Conclusions}

We succeeded in preparing nanostructured NP-NC gels at ambient temperature; these gels are composed of well-defined mono and bimetallic NPs that are strongly immobilized within the polymer-clay network. The metal ions initially interact with the silanol groups of the CNSs during the swelling of the NC 
gel and are then slowly reduced to the corresponding metal NPs near to the CNSs. This trapping process means that the NPs do not move out of the NC gel matrix, even upon repeated swelling and deswelling in water. Furthermore, the present work revealed the new possibility of preparing stable hybrid NP-NCgel materials that exhibit excellent catalytic properties, thereby further expanding the scope of their applications in many advanced research fields. This novel combination of nanoparticles and mechanically tough NC gels opens up many new functionalnanomaterial design possibilities for their widespread use in a variety of reactions and applications.

\section{Acknowledgements}

DV thanks Ahmedabad University and Department of Science and Technology-The Science \& Engineering Research Board (ECR/2016/001210) for financial support. This work as also supported by JSPS KAKENHI grant number 18K05242. The authors thank the DIC Analysis Center (DIC Corp. Japan) for the TEM, and XRD analyses.

\section{References}

[1] Haraguchi K., Takehisa T.: Nanocomposite hydrogels: A unique organic-inorganic network structure with extraordinary mechanical, optical, and swelling/de-swelling properties. Advanced Materials, 14, 1120-1124 (2002). https://doi.org/10.1002/15214095(20020816)14:16<1120::AID-ADMA1120>3.0.CO;2-9

[2] Haraguchi K., Li H-J., Matsuda K., Takehisa T., Elliott E.: Mechanism of forming organic/inorganic network structures during in-situ free-radical polymerization in PNIPA-clay nanocomposite hydrogels. Macromolecules, 38, 3482-3490 (2005).

https://doi.org/10.1021/ma047431c

[3] Haraguchi K.: Synthesis and properties of soft nanocomposite materials with novel organic/inorganic network structures. Polymer Journal, 43, 223-241 (2011). https://doi.org/10.1038/pj.2010.141

[4] Wang Q., Mynar J. L., Yoshida M., Lee E., Lee M., Okuro K., Kinbara K., Aida T.: High-water-content mouldable hydrogels by mixing clay and a dendritic molecular binder. Nature, 463, 339-343 (2010).

https://doi.org/10.1038/nature08693

[5] Yao C., Liu Z., Yang C., Wang W., Ju X-J., Xie R., Chu L-Y.: Poly( $N$-isopropylacrylamide)-clay nanocomposite hydrogels with responsive bending property as temperature-controlled manipulators. Advanced Functional Materials, 25, 2980-2991 (2015).

https://doi.org/10.1002/adfm.201500420
[6] Haraguchi K., Takada T., Haraguchi R.: Nanocomposite gels by initiator-free photopolymerization: Role of plasma-treated clay in the synthesis and network formation. ACS Applied Nano Materials, 1, 418-425 (2018).

https://doi.org/10.1021/acsanm.7b00264

[7] Haraguchi K., Uyama K., Tanimoto H.: Self-healing in nanocomposite hydrogels. Macromolecular Rapid Communication, 32, 1253-1258 (2011).

https://doi.org/10.1002/marc.201100248

[8] Haraguchi K., Kimura Y., Shimizu S.: Reversible generation of large retractive tensile forces in isometric chemo-mechanical actuators composed of nanocomposite hydrogels and aqueous $\mathrm{NaCl}$ solutions. Soft Matter, 14, 927-933 (2018).

https://doi.org/10.1039/c7sm01935a

[9] Haraguchi K., Murata K., Takehisa T.: Stimuli-responsive nanocomposite gels and soft nanocomposites consisting of inorganic clays and copolymers with different chemical affinities. Macromolecules, 45, 385-391 (2012).

https://doi.org/10.1021/ma202114z

[10] Ning J., Li G., Haraguchi K.: Synthesis of highly stretchable, mechanically tough, zwitterionic sulfobetaine nanocomposite gels with controlled thermosensitivities. Macromolecules, 46, 5317-5328 (2013).

https://doi.org/10.1021/ma4009059

[11] Liu M., Ishida Y., Ebina Y., Sasaki T., Aida T.: Photolatently modulable hydrogels using unilamellar titania nanosheets as photocatalytic crosslinkers. Nature Communication, 4, 3029/1-3029/7 (2013).

https://doi.org/10.1038/ncomms3029

[12] Haraguchi K., Varade D.: Platinum-polymer-clay nanocomposite hydrogels via exfoliated clay-mediated in situ reduction. Polymer, 55, 2496-2500 (2014).

https://doi.org/10.1016/j.polymer.2014.03.040

[13] Talapin D. V., Lee J-S., Kovalenko M. V., Shevchenko E. V.: Prospects of colloidal nanocrystals for electronic and optoelectronic applications. Chemical Reviews, 110, 389-458 (2012).

https://doi.org/10.1021/cr900137k

[14] Daniel M-C., Astruc D.: Gold nanoparticles: Assembly, supramolecular chemistry, quantum-size-related properties, and applications toward biology, catalysis, and nanotechnology. Chemical Reviews, 104, 293-346 (2004).

https://doi.org/10.1021/cr030698+

[15] Sahiner N.: Soft and flexible hydrogel templates of different sizes and various functionalities for metal nanoparticle preparation and their use in catalysis. Progress in Polymer Science, 38, 1329-1356 (2013).

https://doi.org/10.1016/j.progpolymsci.2013.06.004

[16] Sahiner N., Ozay O., Aktas N., Inger E., He J.: The on demand generation of hydrogen from Co-Ni bimetallic nano catalyst prepared by dual use of hydrogel: As template and as reactor. International Journal of Hydrogen Energy, 36, 15250-15258 (2011). https://doi.org/10.1016/j.ijhydene.2011.08.082 
[17] Sahiner N., Butun S., Ozay O., Dibek B.: Utilization of smart hydrogel-metal composites as catalysis media. Journal of Colloid and Interface Science, 373, 122-128 (2012).

https://doi.org/10.1016/j.jcis.2011.08.080

[18] Sahiner N., Sagbas S.: The preparation of poly(vinyl phosphonic acid) hydrogels as new functional materials for in situ metal nanoparticle preparation. Colloids and Surfaces A: Physicochemical and Engineering Aspects, 418, 76-83 (2013).

https://doi.org/10.1016/j.colsurfa.2012.11.026

[19] Adhikari B., Biswas A., Banerjee A.: Graphene oxidebased hydrogels to make metal nanoparticle-containing reduced graphene oxide-based functional hybrid hydrogels. ACS Applied Materials and Interfaces, 4, 5472 5482 (2012).

https://doi.org/10.1021/am301373n

[20] Kang C., Wang L., Bian Z., Guo H., Ma X., Qiu X., Gao L.: Supramolecular hydrogels derived from cyclic amino acids and their applications in the synthesis of Pt and Ir nanocrystals. Chemical Communications, 50, 13979-13982 (2014).

https://doi.org/10.1039/C4CC06419D
[21] Varade D., Haraguchi K.: Synthesis of highly active and thermally stable nanostructured Pt/clay materials by clay-mediated in situ reduction. Langmuir, 29, 19771984 (2013). https://doi.org/10.1021/la3044945

[22] Narayanamoorthy B., Datta K. K. R., Eswaramoorthy M., Balaji S.: Improved oxygen reduction reaction catalyzed by Pt/clay/nafion nanocomposite for PEM fuel cells. ACS Applied Materials and Interfaces, 4, 36203626 (2012). https://doi.org/10.1021/am300697q

[23] Zhang W., Li M. K. S., Yue P-L., Gao P.: Exfoliated Ptclay/Nafion nanocomposite membrane for self-humidifying polymer electrolyte fuel cells. Langmuir, 24, 2663-2670 (2008) https://doi.org/10.1021/la702153v

[24] Zhang R., Hummelgård M., Olin H.: Simple synthesis of clay-gold nanocomposites with tunable color. Langmuir, 26, 5823-5828 (2010).

https://doi.org/10.1021/la903747v 